Arroyo González, Rosario (2013). Descripción de procesos en la composición escrita de estudiantes universitarios para un desarrollo multilingüe y tecnológico. Revista de Investigación Educativa, 31 (1), 167-184.

http://dx.doi.org/10.6018/rie.31.1.148441

\title{
DESCRIPCIÓN DE PROCESOS EN LA COMPOSICIÓN ESCRITA DE ESTUDIANTES UNIVERSITARIOS PARA UN DESARROLLO MULTILINGÜEYTECNOLÓGICO
}

\author{
Dra. Rosario Arroyo González \\ Universidad de Granada
}

\section{RESUMEN}

La finalidad de esta investigación es describir los procesos cognitivos-lingüísticos, metacognitivo-afectivos y socioculturales implicados en la composición escrita de estudiantes universitarios, procesos que de forma universal son aplicados por lo escritores expertos en cualquier lengua, por esto se pueden considerar procesos de desarrollo escritor multilingüe. Además, estos procesos son básicos en el uso de la comunicación tecnológica. Para lograr ese fin se aplica un diseño metodológico en el que se aplica una entrevista cognitiva escrita a una muestra de 86 estudiantes universitarios. El método de Análisis de Contenido permite aplicar un sistema de categorías que define operativamente los procesos escritores expresados por los estudiantes. En base al recuento de frecuencias se destaca que los estudiantes universitarios analizados muestran cierto nivel de competencia procedimental, afectiva y sociocultural de la escritura, y se evidencia la necesidad de diseñar programas para lograr un uso profesional y científico de la misma, que insista en procesos condicionales.

Palabras claves: escritura; investigación cualitativa; enseñanza científica superior; procesos de la escritura.

Correspondencia:

Dra. Rosario Arroyo González. Departamento de Didáctica y Organización Escolar. Facultad de Ciencias de la Educación. Universidad de Granada. E-mail: rarroyo@ugr.es 


\title{
DESCRIPTION OF THE PROCESS OF WRITTEN COMPOSITION OF UNIVERSITY STUDENTS FOR A MULTILINGUAL AND TECHNOLOGICAL DEVELOPMENT
}

\begin{abstract}
The purpose of this investigation is to describe the cognitive-linguistic, metacognitveaffective, and socio-cultural processes involved in written composition of university students. These processes are universally applied by expert writers in any language, thus may be considered developmental processes in the multilingual writer. Furthermore, these processes are essential in technological communication. A methodological design was used in this study, in which written cognitive interviews were conducted with a sample of 86 university students. The Method of Content Analysis allowed the application of a categorization system which operatively defined the written processes expressed by students. In terms of frequency, the results showed that university students display a certain degree of procedural, affective and socio-cultural writing competence, and that there is a need for specific programmes which foster professional and scientific use of written composition stressing the importance of conditional processes.
\end{abstract}

Keyword: Writing, Qualitative Research, Scientific Higher Education; Writing Processes.

\section{INTRODUCCIÓN: LA ESCRITURA EN LA CIUDADANÍA DEL SIGLO XXI}

El proceso investigador que se describe en este artículo, se sitúa en el proyecto del Grupo de Investigación EDINVEST (HUM356) titulado “Comunicación escrita multilingüe y tecnologías", bajo la subvención por la Junta de Andalucía e, igualmente, está financiado por el Vicerrectorado de Planificación Calidad y Evaluación Docente de la Universidad de Granada (MVG/PI2007).

Esta investigación, describe los procesos escritores desde un modelo explicativo global de la escritura (Autor \& Hunt, 2010), que se fundamenta en la revisión de todas las líneas de investigación en la escritura.

El modelo mencionado integra los procesos escritores lingüísticos, cognitivos, metacognitivos y socioculturales (MacArthur, Graham \& Fitzgerald, 2006; Bear, Myhill, Nystrand \& Riley, 2009; Myhill \& Fisher, 2010). A su vez fundamenta la enseñanza de la escritura, usando tecnologías informatizadas (Cabero \& Llorente, 2007) en cualquier nivel educativo y de forma multilingüe (Canagarajah \& Jerskey, 2009), con sus correspondientes adaptaciones (Autor, 2009). Por lo tanto, en este estudio se analiza todos los elementos que inciden en la escritura.

Destacado el interés de la investigación en la enseñanza de la escritura, la aportación novedosa de este artículo, consiste en la descripción de los procesos cognitivos-lingüísticos, metacognitivos-afectivos y socioculturales que expresan estudiantes universitarios. Sin duda, conocer el nivel de conocimiento de dichos procesos en estudiantes universitarios, es el punto de partida para el diseño de programas educativos que promueva el desarrollo de la escritura, adecuándose a las nuevas exigencias profesionales de las Sociedades del Siglo XXI y a los niveles de aprendizaje en los mismos. 


\section{REVISIÓNTEÓRICA DETODOS LOS PROCESOS DE DESARROLLO ESCRI- TOR}

Entre los años 40 y 70, el interés investigador por la escritura se centra en los procesos lingüísticos del texto (Rentel \& King, 1983; Fayol, 1991) prestando especial atención a los aspectos sintácticos y léxicos. Posteriormente, se empiezan a desarrollar modelos escritores centrados en los procesos cognitivos de la escritura. Estos son: procesos de planificación, de transcripción, de revisión y de metacognición de la escritura (Flower \& Hayes, 1981; Scardamalia \& Bereiter 1983; De Beaugrande, 1984). A partir de los años 80 el interés de la investigación se ocupa de los procesos afectivos que intervienen en la escritura tales como: la concentración, la motivación, el autoconcepto (Daly \& Wilson, 1983; Dipardo \& Schnack, 2004; Bruning \& Horn, 2000; Harris, Graham, Mason \& Saddler, 2002; Fidalgo, Arias-Gudin, García \& Torrance, 2010).

Desde los años 90, han surgido investigaciones interesadas por el desarrollo social y cultural de la escritura (Sperling, 1996; Freedman, 1996; Nystrand, 2006; Schultz \& Fecho, 2000), donde se identifican importantes diferencias en la funcionalidad e interpretación comunicativa de los contenidos y de los mensajes que se transmiten. Dentro de estos estudios socioculturales sobre la escritura, se muestra el lenguaje escrito como un potente instrumento de expresión de valores personales y comunitarios (Autor, 2000a, 2000b, 2001). Otro campo de interés sociocultural de los últimos años, viene siendo la promoción de las tecnologías informatizadas en la enseñanza de la escritura (Goldberg, Russell \& Cook, 2003; MacArthur, 2006), tanto, para apoyar los aprendizajes de procesos y habilidades propios de la escritura, como para crear nuevas formas, soportes y contextos en la escritura. Por último, las investigaciones sobre el desarrollo escritor en una segunda lengua o de forma multilingüe (Krapels, 1990; Fitzgerald, 2006; Ball, 2006; Matsuda, Ortmeier-Hooper \& Matsuda, 2009, destacan: a) la influencia de los profesores, la clase y el contexto comunitario en la enseñanza de la escritura, b) la influencia de la cultura del alumno o el discurso primario de su familia en el estilo de su escritura; y c) la utilización de procesos comunes de la escritura en diferentes lenguas.

En perspectiva global, el desarrollo de la composición escrita no es posible sin una conceptualización de la misma, que descubra la incardinación de procesos cognitivoslingüísticos, metacognitivos-afectivos y procesos socioculturales. Pero esta conceptualización de la composición escrita exige procesos de reflexividad sobre el desarrollo de la composición escrita (Autor, 2006b; Kostouli, 2009; Moss, 2009):

En definitiva, la integración de procesos en el desarrollo escritor, significa entender que el desarrollo de la composición escrita es fruto de una diversidad de procesos, tanto metacognitivos (que incluye lo lingüístico, cognitivo y afectivo) como socioculturales y que entre estos existen núcleos de conexión, y de éstos con el texto (Autor \& Hunt, 2011).

La gran innovación de esta investigación consiste en operativizar cada una de estas dimensiones escritoras con el fin de describir todos los procesos que se aplican en la composición escrita, basándose en una muestra de estudiantes universitarios. 


\section{OBJETIVOS DE LA INVESTIGACIÓN}

La finalidad de esta investigación es describir todos los procesos cognitivos-lingüísticos, metacognitvo-afectivos y socioculturales implicados en la composición escrita de una muestra de estudiantes universitarios. Para lograr este propósito se persiguen diferentes objetivos:

1. Elaborar y aplicar una entrevista cognitiva y un sistema de categorías para recoger información sobre esos procesos, en una muestra de estudiantes universitarios.

2. Identificar macro-procesos y procesos escritores en esa muestra de estudiantes universitarios

3. Definir operativamente cada uno de los procesos que intervienen en el proceso escritor de los estudiantes universitarios analizados

4. Describir las operaciones escritoras que esos estudiantes realizan en los procesos escritores con altas frecuencias proporcionales

5. Identificar las operaciones y procesos escritores de baja frecuencia proporcional en la muestra de estudiantes mencionada

6. Establecer líneas de estrategia didáctica para el desarrollo global de la composición escrita que respondan a las exigencias tecnológicas y multilingües de la ciudadanía

Estos objetivos han guiado cada una de las fases del proceso investigador que se describe en este artículo.

\section{MÉTODO}

Los objetivos de esta investigación son, esencialmente descriptivos y para su logro se han aplicado las siguientes estrategias, procedimientos e instrumentos:

\section{I. Contexto y muestra}

En contexto en el que se realiza esta investigación es la Facultad de Ciencias de la Educación de la Universidad de Granada. Se aplicó el muestreo casual (Mayorga \& Ruiz, 2002), es decir, participación voluntaria de los sujetos de 86 estudiantes de primer curso, de la Titulación de Maestro. La muestra resultante, estuvo compuesta en un $74 \%$ por mujeres y en un $26 \%$ por hombres. El $35 \%$ de la muestra tenía una edad de 18 años, el $40 \%$ de 19 años y para el 20\% restante la edad podía oscilar entre 23 y 27 años.

\subsection{Procedimientos e Instrumentos}

Para la selección de la muestra, se ofertó a los estudiantes un seminario con la finalidad de aprender a escribir textos argumentativos científicos. Los estudiantes se adscribieron libremente a este seminario que fue impartido por profesores de universidad como créditos prácticos de sus asignaturas. Una de las actividades de este seminario fue la evaluación de procesos escritores.

En base a las entrevistas cognitivas elaboradas y aplicadas, en la investigaciones de Grahan \& Harris, (2005a:143) y del Grupo EDINVEST (Salvador \& García, 2005:67), se 
elaboró una entrevista cognitiva escrita en la que se interroga al estudiante sobre sus propios procesos escritores (Autor, 2009: 272), según el Modelo Metasociocognitivo de Desarrollo Escritor (Autor, 2009; Autor \& Hunt, 2011). Se trata de una entrevista semiestructurada con 37 preguntas del tipo: Mientras escribes el texto ¿utilizas nexos y/o expresiones para unir frases, párrafos...? ¿Qué tipo de nexos? Pon algún ejemplo. Esta entrevista se aplicó después de realizar la tarea de escribir un texto. Los estudiantes tuvieron una hora para su realización, en horas lectivas y en las aulas asignadas. El profesor estuvo en todo momento presente para solucionar cualquier duda que surgiese en el transcurso de la tarea.

Para el análisis de los datos generados tras la aplicación de la mencionada entrevista, se aplicó el Método de Análisis de Contenido (Gubrium \& Holstein, 2000; Autor, 2000b). En el proceder de este método se estableció:

1. El universo a analizar, compuesto por todas las entrevistas aplicadas a los distintos sujetos de la muestra.

2. Las unidades de contexto, constituidas por la información registrada en cada entrevista.

3. Las unidades de registro, esto es una palabra, una frase o un párrafo, siempre que aportasen información completa sobre alguna operación escritora.

4. Elaboración del sistema de categorías teórico fundamentado en la revisión teórica (Tabla 1).

TABLA 1

SISTEMA DE CATEGORÍAS DE LOS PROCESOS ESCRITORES

\begin{tabular}{|l|l|l|}
\hline & Categorías de Procesos Escritores & Códigos \\
\hline 1 & Planificación de la escritura & Sp \\
2 & Transcripción de la escritura & St \\
3 & Revisión de la escritura & Sr \\
5 & Teoría de la Tarea escritora & Sta \\
6 & Teoría del Texto escrito & Stx \\
7 & Autorregulación de la escritura & Sarr \\
8 & Motivación para la escritura & Sac \\
9 & Creatividad en la escritura & Sm \\
10 & Condicionamiento Político en la escritura & Sc \\
11 & Condiciones Comunitarias y/o Profesionales en la escritura & Ss \\
12 & Construcción de la Identidad en la escritura & Scp \\
\hline
\end{tabular}

La aplicación del Método de Análisis de Contenido ha pasado por una serie de fases:

Fase 1.- Exploración de las unidades de contexto y clasificación de la información en base al sistema de categorías (Tabla 1).

Fase 2.- Codificación de todas las entrevistas (universo) según los códigos asignados en el Sistema de Categorías.

Fase 3.- Definición de cada categoría, de forma emergente, en función de las operaciones escritoras identificadas en las respuestas de los estudiantes entrevistados (Tabla 2). 
TABLA 2

DEFINICIÓN OPERATIVA DE LOS PROCESOS ESCRITORES

\begin{tabular}{|c|c|c|}
\hline & \begin{tabular}{|l} 
Procesos Escritores \\
\end{tabular} & Operaciones que se realizan en cada proceso \\
\hline 1 & $\begin{array}{l}\text { Proceso } \\
\text { procedimental/ } \\
\text { Declarativo. } \\
\text { Planificación de la } \\
\text { escritura }\end{array}$ & $\begin{array}{l}\text { 1. Pensar en el auditorio a quien se dirige el escrito } \\
\text { 2. Pensar en los objetivos del escrito } \\
\text { 3. Generar de ideas según el tema del texto } \\
\text { 4. Seleccionar de ideas más adecuadas al tema } \\
\text { 5. Ordenar las ideas según la estructura del texto } \\
\text { 6. Buscar fuentes donde encontrar más ideas } \\
\text { 7. Estrategias para registrar todo lo planificado }\end{array}$ \\
\hline 2 & $\begin{array}{l}\text { Proceso } \\
\text { procedimental/ } \\
\text { Declarativo. } \\
\text { Transcripción de la } \\
\text { escritura }\end{array}$ & $\begin{array}{l}\text { 8. Aplicar una estructuras de escritura gramatical } \\
\text { 9. Aplicar mecanismos de progresión y coherencia textual } \\
\text { 10. Utilizar un vocabulario concreto para el tema del texto } \\
\text { 11. Seleccionar las palabras más adecuadas al estilo del texto } \\
\text { 12. Aplicar la ortografía y grafía adecuada } \\
\text { 13. Utilizar los soportes tecnológicos y programas adecuados }\end{array}$ \\
\hline 3 & $\begin{array}{l}\text { Proceso } \\
\text { procedimental/ } \\
\text { Declarativo. Revisión } \\
\text { de la escritura }\end{array}$ & $\begin{array}{l}\text { 14. Adecuar el texto escrito al texto planificado } \\
\text { 15. Modificar la estructura y léxico de la oración } \\
\text { 16. Cambiar partes del texto para lograr la unidad global } \\
\text { 17. Modificar signos de puntuación, ortografía y caligrafía } \\
\text { 18. Aplicar estrategias de revisión por otros } \\
\text { 19. Aplicar estrategias de revisión por uno mismo }\end{array}$ \\
\hline 4 & $\begin{array}{l}\text { Proceso } \\
\text { procedimental. Teoría } \\
\text { de la Tarea escritora }\end{array}$ & $\begin{array}{l}\text { 20. Conocer las funciones socioculturales del texto } \\
\text { 21. Conocer las características socioculturales del auditorio } \\
\text { 22. Conocer la relación entre las funciones y el auditorio del } \\
\text { texto; y la forma, el contenido, la estructura, el código lingüís- } \\
\text { tico y el soporte del texto } \\
\text { 23. Conocer estrategias para escribir un buen texto }\end{array}$ \\
\hline 5 & $\begin{array}{l}\text { Proceso condicional. } \\
\text { Teoría del Texto }\end{array}$ & $\begin{array}{l}\text { 24. Conocer la relación entre el tipo de texto (objetivos, conten- } \\
\text { dido, forma) y el código lingüístico } \\
\text { 25. Conocer la relación entre el tipo de texto (objetivos, conten- } \\
\text { dido, forma) y el tipo de soporte } \\
\text { 26. Conocer la relación entre el tipo de texto (objetivos, conten- } \\
\text { dido, forma) y el tipo de estructura } \\
\text { 27. Clasificar los párrafos y partes del texto según criterios de } \\
\text { jerarquía y unidad }\end{array}$ \\
\hline 6 & $\begin{array}{l}\text { Proceso condicional. } \\
\text { Autorregulación de la } \\
\text { escritura }\end{array}$ & $\begin{array}{l}\text { 28. Seleccionar las estrategias y el código lingüístico según el } \\
\text { tipo de texto (objetivos, contendido, forma) } \\
\text { 29. Aplicar autoinstrucciones en el proceso escritor } \\
\text { 30. Reflexiónar sobre las propias competencias escritoras }\end{array}$ \\
\hline
\end{tabular}




\begin{tabular}{|l|l|l|}
\hline & Procesos Escritores & \multicolumn{1}{|c|}{ Operaciones que se realizan en cada proceso } \\
\hline 7 & $\begin{array}{l}\text { Proceso afectivo. } \\
\text { Autocontrol de la } \\
\text { escritura }\end{array}$ & $\begin{array}{l}\text { 31. Seleccinar el sentimiento adecuado al tipo texto (objetivos, } \\
\text { contendido, forma) } \\
\text { 32. Lograr la concentración que exige la tarea de escritura }\end{array}$ \\
\hline 8 & $\begin{array}{l}\text { Proceso afectivo. } \\
\text { Motivación hacia la } \\
\text { escritura }\end{array}$ & $\begin{array}{l}\text { 33. Disponer de recursos humanos y materiales de apoyo en la } \\
\text { escritura } \\
\text { 34. Desarrollar un autoconcepto positivo en las tareas de la es- } \\
\text { critura }\end{array}$ \\
\hline 9 & $\begin{array}{l}\text { Proceso afectivo. } \\
\text { Creatividad en la } \\
\text { escritura }\end{array}$ & $\begin{array}{l}\text { 35. Expresar valores propios en la escritura } \\
\text { 36. Aplicar estrategias propias en la escritura } \\
\text { 37. Desarrollar estilos de expresión escrita propios }\end{array}$ \\
\hline 10 & $\begin{array}{l}\text { Proceso sociocultural. } \\
\text { pondicionamien-to en la escritura }\end{array}$ & $\begin{array}{l}\text { 38. Conocer los condicionamientos político-económicos sobre } \\
\text { la propia escritura (soportes, idioma, temas, géneros, utilidad } \\
\text { de mis textos) } \\
\text { 39. Conocer las funciones sociales de la propia escritura (mis } \\
\text { escritos mejoran la sociedad) }\end{array}$ \\
\hline 11 & $\begin{array}{l}\text { Proceso sociocultural. } \\
\text { Condiciones } \\
\text { comunitarias y/o } \\
\text { profesionales en la } \\
\text { escritura }\end{array}$ & $\begin{array}{l}\text { 40. Usar la escritura en contextos cotidianos y/o profesionales } \\
\text { 41. Lograr intereses comunitarios y/o profesionales en los usos } \\
\text { cotidianos de la propia escritura (mis escritos sirven para cono- } \\
\text { cer o ayudar a los demás) } \\
\text { 42. Usar códigos lingǘsticos diferentes en los escritos cotidia- } \\
\text { nos y/o profesionales } \\
\text { 43. Construir textos de forma colaborativa }\end{array}$ \\
\hline 12 & $\begin{array}{l}\text { Proceso sociocultural. Usar la escritura para expresar experiencias o ideas propias } \\
\text { Construcción de } \\
\text { la Identidad en la } \\
\text { escritura }\end{array}$ & $\begin{array}{l}\text { 45. Usar de forma competente la lengua escrita materna y otras } \\
\text { lenguas } \\
\text { 46. Lograr intereses propios en los uso de la escritura }\end{array}$ \\
\hline
\end{tabular}

Fase 4.- Recuento de frecuencias directas, es decir, número de veces que aparece cada código, referido a cada procesos, en todos los sujetos de la muestra y su traducción a porcentajes (Tabla 3, Tabla 4)

El procedimiento básico usado para garantizar la fiabilidad cualitativa es "la triangulación" (Denzin, 1970). Todos los documentos, es decir, todas las entrevistas fueron codificadas aplicando el sistema de categorías (Tabla 1), de forma simultánea, por tres investigadores llegando a un coeficiente de concordancia de $100 \%$ para todas las categorías. El universo de datos recogido fue de 86 entrevistas cognitivas, que tras su análisis arrojó una frecuencia total de 7038 operaciones codificadas en dicha entrevistas. Por lo tanto, estas frecuencias directas constituyen una cantidad suficiente de datos para llegar a conclusiones fiables.

Para garantizar la validez de los instrumentos de recogida y análisis de los datos, el sistema de categorías y la entrevista fue sometido al Juicio de 5 Expertos. Para ello se creó un grupo de discusión en el que se aplico la reflexividad (Bordieu, 2003), sobre un cuestionario que valoraba los instrumentos mencionados, siguiendo los criterios de Fox (1981) (ver Tabla, 3) 
TABLA 3

CRITERIOS PARA VALIDAR LA ENTREVISTA COGNITIVA Y EL SISTEMA DE CATEGORÍAS DE LA ESCRITURA

\begin{tabular}{|l|l|}
\hline Exhaustividad & $\begin{array}{l}\text { ¿El item de la entrevista recoge información sobre la totalidad de una } \\
\text { operación escritora? ¿Las operaciones del sistema permiten agotar el } \\
\text { contenido de la totalidad del proceso escritor? }\end{array}$ \\
\hline $\begin{array}{l}\text { Exclusión } \\
\text { mutua }\end{array}$ & $\begin{array}{l}\text { ¿El item de la entrevista recaba información sobre operaciones ecritoras } \\
\text { diferentes a los de otros items? ¿Las operaciones del sistema no se repiten } \\
\text { en otros procesos? }\end{array}$ \\
\hline Homogeneidad & $\begin{array}{l}\text { ¿Los items de la estrevista y las operaciones del sitema están formuladas } \\
\text { de acuerdo con los modelos de desarrollo escritor? }\end{array}$ \\
\hline Pertinencia & $\begin{array}{l}\text { ¿La formulación del item de la entrevista está adaptado al nivel de } \\
\text { comprensión de los estudiantes universitarios? ¿La descripción de cada } \\
\text { suprocesos del sistema responde a posibles subprocesos de desarrollo } \\
\text { escritor adquiridos por los estudiantes universitarios? }\end{array}$ \\
\hline Productividad & $\begin{array}{l}\text { ¿Los items de la entrevista facilita la obtención de información rica y } \\
\text { variada sobre una operación escritora? ¿Las operaciones del sistema } \\
\text { ofrecen información ríca y variada sobre el subproceso escritor en cuestión? }\end{array}$ \\
\hline
\end{tabular}

Con el propósito de analizar el grado de acuerdo entre los jueces expertos se ha aplicado W de Kendall. Los resultados obtenidos muestran un grado de acuerdo moderado en exhaustividad, exclusión mutua, pertinencia y productividad en cada proceso. En las dimensiones exhaustividad y productividad se observa los mayores niveles de acuerdo siendo más bajo en homogeneidad. En cualquiera caso las valoraciones de los jueces expertos en todas las dimensiones analizadas han sido positivas.

\section{RESULTADOS}

Una aproximación extensiva a los procesos expresados por los sujetos de la muestra, permite detectar sus rangos de preferencia con respecto a los macro-procesos y procesos escritores. Observando la Tabla 4, se comprueba que cada uno de los procesos escritores expresados por los sujetos de la muestra, se pueden agrupar en macro-procesos.

TABLA 4

FRECUENCIAS DIRECTAS Y PROPORCIONALES DE MACRO-PROCESOS ESCRITORES

\begin{tabular}{|l|c|c|c|c|c|}
\hline & $\begin{array}{c}\text { SD } \\
\text { declarativos }\end{array}$ & $\begin{array}{c}\text { SC } \\
\text { condicionales }\end{array}$ & $\begin{array}{c}\text { SA } \\
\text { afectivos }\end{array}$ & $\begin{array}{c}\text { SSC } \\
\text { socioculturales }\end{array}$ & Total \\
\hline Frecuencia & 3204 & 1196 & 1166 & 1472 & 7038 \\
$\%$ & $45.52 \%$ & $16.99 \%$ & $16.56 \%$ & $20,91 \%$ & $100 \%$ \\
\hline
\end{tabular}

Observando el Gráfico 1, se aprecia que los macro-procesos de conocimiento declarativo/procedimental (SD) sobre la escritura, acumulan el 45,52\%. Esto significa que los sujetos de la muestra realizan, sobre todo, procesos de planificación, transcripción y revisión del texto. 


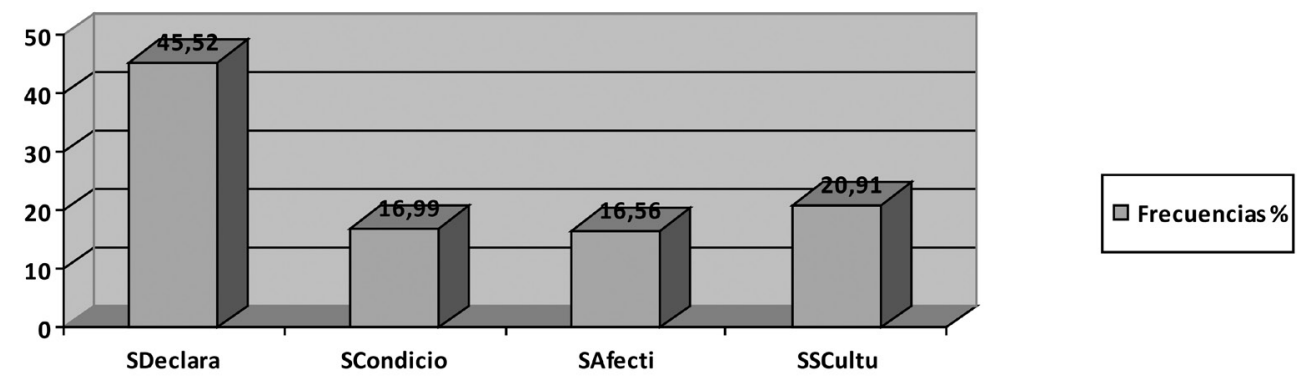

GRÁFICO 1

FRECUENCIAS PROPORCIONALES DE MACRO-PROCESOS ESCRITORES

Los procesos de conocimiento condicional (SC) y afectivos (SA) acumulan un $16.99 \%$ y un $16.56 \%$, respectivamente. Por lo tanto, las estrategias escritoras de Teoría de la Tarea, de Teoría del Texto y de Autorregulación de la escritura tienen el mismo peso, para los sujetos de la muestra, que: a) las estrategias de Autocontrol de la emociones en la escritura, b) las estrategias de Motivación hacia la escritura; y c) las estrategias de Creatividad de los propios texto. Por último, los procesos socioculturales de la composición escrita suponen el 20,91\%. Esto significa que los sujetos de la muestra, verbalizan por escrito aspectos políticos, comunitarios/profesionales y de construcción de su propia identidad; y entienden que estos aspectos influyen en el contenido y forma de sus textos escritos.

Observando la Tabla 5 y el Gráfico 2, se puede hacer un estudio más profundo de los procesos escritores expresados por los sujetos de la muestra.

TABLA 5

FRECUENCIAS DIRECTAS Y PROPORCIONALES DE PROCESOS ESCRITORES

\begin{tabular}{|c|c|c|c|c|c|c|c|c|c|c|c|c|c|}
\hline & Sp & St & Sr & Sta & Stx & Sarr & Sac & Sm & Sc & Ss & Scp & Si & PD/\% \\
\hline PD & 1530 & 1106 & 568 & 356 & 336 & 504 & 17 & 784 & 368 & 158 & 764 & 550 & 7038 \\
\hline$\%$ & $21.73 \%$ & $15.71 \%$ & $8.07 \%$ & $5.05 \%$ & $4.77 \%$ & $7,16 \%$ & $0.19 \%$ & $11.13 \%$ & $5.22 \%$ & $2,24 \%$ & $10.85 \%$ & $7.81 \%$ & $100 \%$ \\
\hline
\end{tabular}

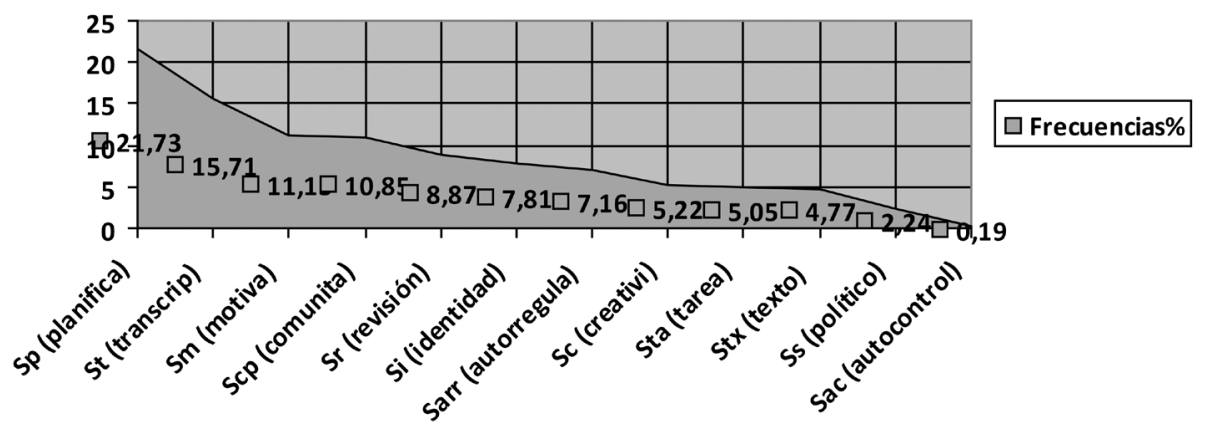

GRÁFICO 2

FRECUENCIAS PROPORCIONALES DE PROCESOS ESCRITORES 
En primer lugar, los procesos más frecuentes son los procesos de Planificación (Sp) de la escritura, con un 21,73\%. Le siguen los procesos de Transcripción con un 15,71\%. Ambos pertenecen al macro-proceso procedimental/declarativo de la escritura.

En segundo lugar, destacan los procesos de Motivación (Sm) con un 11,13\% y los procesos Comunitarios/Profesionales (Scp) con un 10,85\%. Ambos pertenecen a los macro-procesos afectivos y socioculturales, respectivamente.

En tercer lugar y próximos al 10\% se encuentran, los procesos de Revisión (Sr: 8,07\%); los procesos de Identidad (Si: 7,81\%) y los procesos de Autorregulación (Sarr: 7,16\%). El proceso con menor frecuencia proporcional, pertenece al macro-proceso de los conocimientos condicionales.

Por último en el 5\%, o en inferior porcentaje, se identifican los procesos de Creatividad (Sc: 5,22\%), de Teoría de la Tarea (Sta: 5,05\%); de Teoría del Texto (Stx: 4,77\%), Políticos (Sc: 2,24\%) y de Autocontrol (Sac: 0,19\%). Así pues los procesos con menos frecuencia pertenecen al macro-proceso de conocimiento condicional, afectivo y sociocultural.

Seguidamente se describirán los procesos con más frecuencia proporcional. Para ello se utilizan citas textuales sacadas de las entrevistas aplicadas a los sujetos de la muestra. Cada cita tiene un código $(E x, P n)$ que se refiere a la entrevista (Ex) en la que aparece la cita y la pregunta $(\mathrm{Pn})$ con la que se corresponde.

\section{I. Operaciones de Planificación de la escritura}

Dentro de los procesos de Planificación, los sujetos de la muestra admiten, sobre todo, realizar operaciones para ordenan las ideas que van a expresar en su texto: "Escribo una pequeña síntesis, un esquema en el que las ideas principales se vean reflejadas y que me permita ampliarlo con las ideas secundarias" (E30, P4). Además, reconocen que esa forma de ordenar las ideas viene dada por el género literario del texto: "No es lo mismo escribir una carta que un cuento o el resumen de un texto..." (E60, P22).

Otras operaciones de Planificación, que se realiza con alta frecuencia, es consultar fuentes de ideas para escribir el texto: "Suelo buscar sobre todo en Internet, pero también consulto algunos libros que tengan relación con el tema a tratar" (E02, P5). También, los universitarios piensan en los objetivos de su texto, que suelen ser de carácter académico "...ya que si es mandado lo hago como cuestión de trabajo, y si es para mí, es una forma de deshago..." (E37, P19); aunque también muestran objetivos personales: "Escribiendo por placer expongo emociones y sensaciones..." (E70, P3.). Les sigue en frecuencia la operación de generar ideas para escribir el texto: “...pienso y leo con objeto de encontrar algunas ideas que me puedan ser útiles a la hora de escribir un texto" (E29, P5); y la operación de considerar la persona que va a leer el texto: "Pienso que si va destinado al profesor o para una publicación tendrá que ser mucho más elaborado que si lo escribo para un amigo" (E23, P20).

Por último, en frecuencias mucho más bajas se encuentran las operaciones de registras ideas: "En un borrador voy apuntando lo que creo que tiene mayor importancia" (E9, P6); y la operación de seleccionar ideas: "Busco ideas principales y secundarias" (E50, P4). 


\subsection{Operaciones de Transcripción de la escritura}

Dentro de los procesos de transcripción, los estudiantes de la muestra hacen referencia a su preocupación por la ortografía y grafía: “Tengo bastante cuidado de no cometer errores ortográficos, aplico mucho las tildes, las mayúsculas..." (E43, P11). Reconocen utilizar variedad de soportes y recursos auxiliares como: "Libretas, folios, lápices, ceras, rotuladores, acuarelas, pantalla de ordenador, teclado..." (E8, P34). También, destacan la aplicación de operaciones escritoras para lograr la progresión de las ideas en el texto: “...y las voy [las ideas] relacionando, unas con otras, hasta que decido cómo quiero que vaya quedando el texto" (E4, P4); y la coherencia textual "Utilizo conjunciones ( $y, 0$, pero, como...), preposiciones, $y$ expresiones hechas (sin embargo, como iba diciendo, en consecuencia, es decir, etc.)" (E63, P8).

Por último, con menor frecuencia, se encuentran las operaciones de aplicar una estructura de escritura gramatical: "Cuido la longitud de las oraciones y la coherencia del texto para conseguir una secuencia lógica de ideas" (E14, P26); y de utilizar un vocabulario concreto para el tema del texto: "cuando una palabra veo que se repite mucho en el texto busco sinónimos" (E2, P9)"... las clasifico según a la familia a la que pertenece" (E59, P10). Seleccionan las palabras según el estilo del texto: "...utilizando las palabras que me parecen más adecuadas" (E24, P10).

\subsection{Operaciones de Motivación y Comunitarias/Profesionales en la composición escrita}

Dentro de los procesos motivacionales hacia la escritura destaca el reconocimiento de haber tenido apoyos materiales de carácter estandarizado en su desarrollo escritor: "En internet, diccionarios, por ejemplo en la página web de antónimos y sinónimos españoles". (E74, P9); así como, apoyos humanos: "me ayudaron mi familia, mis maestros" (E17, P29). Esta operación presenta una frecuencia muy alta. Con una frecuencia muy baja, se mencionan las operaciones de autoconcepto en la escritura y, cuando se hace, unas veces es en sentido positivo: "Sólo en contadas ocasiones no termino los textos" (E30, P21) y otras en sentido negativo:"...he aprendido a hablar de esa manera y me cuesta mucho escribir bien esos artículos" (E81, P11).

Con respecto a los procesos Comunitarios/Profesionales en la composición escrita destacan, con frecuencias altas, la operación de lograr intereses comunitarios y/o profesionales en los usos cotidianos de la propia escritura: "Por ejemplo el ensayo que he hecho sobre la enseñanza multilingüe, espero aportarle más información a mi profesora y a mis compañeros dando mis opiniones, mis argumentos" (E53, P19). A esta operación le sigue el uso de la escritura en contextos cotidianos y/o profesionales: ". . he escrito en mi casa" (E75, P31) "...en el instituto, en la facultad" (E18, P36). Con más baja frecuencia se mencionan el uso códigos lingüísticos diferentes: "...he escrito en inglés, español y francés" (E13, P34). También con baja frecuencia se construyen textos de forma colaborativa y, cuando se hace, es por exigencias académicas: "La mayoría de los textos que he escrito cooperando con otras personas han sido para elaborar trabajos para alguna asignatura en la Facultad" (E29, P35). 


\subsection{Operaciones de Revisión, de Identidad y de Autorregulación en la composición escrita}

Sobre el proceso de Revisión de la escritura, los sujetos de la muestra, admiten realizar las siguientes operaciones en el siguiente orden de frecuencias:

1) Modifican la estructura y léxico de la oración: "Después de escribir cambio y quito nexos" (E1, P14)

2) Adecuan el texto escrito al texto planificado: "comprobar que todas las ideas que he seleccionado son las correctas y apropiadas" (E25, P4) "...hago cambios que me ayuden a realizar la estructura del texto que quiero realizar" (E46, P6)

3) Modificar signos de puntuación, ortografía y caligrafía: "Después de escribir añado, quito signos de puntuación" (E8, P16) “...cambio la caligrafia" (E72, P17)

4) Aplican estrategias de revisión por uno mismo: "reviso continuamente el texto para comprobar que está bien redactado" (E3, P18)

5) Aplicar estrategias de revisión por otros: "...si hay alguien cerca, suelo leérselo para que me dé su opinión" (E4, P18)

Y con una frecuencia casi nula, se expresa la operación de cambiar partes del texto para lograr la unidad global: “...modifico o cambio alguna parte para que la lectura no resulte pesada" (E39, P18).

Sobre los procesos de Identidad destaca, la operación de lograr intereses propios en los uso de la escritura: “...me ha servido para aprender a expresarme mejor, para corregir faltas de ortografía” (E21, P30). Con frecuencias mucho más bajas se mencionan las operación de usar la escritura para expresar experiencias o ideas propias: "Sí, por ejemplo, cuando he escrito sobre experiencias vivenciales, me he basado en experiencias que he tenido con otras personas" (E14, P33); y la operación de usar de forma competente la lengua escrita materna y otras lenguas: “...deberé de utilizar la lengua materna que nos une en nuestro país" (E16, P26)

Con respecto a los procesos de Autorregulación de la escritura destacan por su alta frecuencia, la operación de reflexionar sobre las propias competencias escritoras con expresiones, unas veces en sentido positivo: “...no es algo en lo que suela pensar..." (E37, P29); y otras en sentido negativo: "Hago más hincapié en las últimas [puntuación] para que el texto en sí adquiera el sentido que quiera darle..." (E78, P11).

En frecuencias mucho más bajas se expresan las operaciones de aplicar autoinstrucciones en el proceso escritor: "Utilizar un lenguaje correcto sin cometer faltas de ortografía y estableciendo una buena relación entre los contenidos" (E7, P27). Le sigue en baja frecuencia la operación de seleccionar el código lingüístico según el tipo de texto: “...audiencia científica o universitaria utilizo un lenguaje más científico, si es para familiares, amigos/as, es más coloquial y cercano" (E3, P26). Sin embargo no se reconocen la selección de estrategias escritoras según el tipo de texto.

\subsection{Operaciones de Creatividad}

Con respecto a los procesos de Creatividad, destacan la aplicación de estrategias propias en la escritura: "...escribo en un folio aparte y reflexiono sobre ellas" (E17, P4) "buscando información en otros libros, leyéndolo como si lo hubiera escrito otra persona y formulando 
mi punto de vista después sobre lo leído" (E52, P21). Son menos frecuentes las expresiones de valores propios en la escritura y el desarrollar estilos de expresión escrita propios.

\section{DISCUSIÓNY CONCLUSIONES}

En base a los análisis realizados se puede establecer el perfil escritor de los estudiantes universitarios analizados.

En primer lugar, los sujetos analizados, antes de escribir un texto, suelen ordenar sus ideas en principales y secundarias. Consultan en fuentes para buscar ideas. Piensan en los objetivos de sus escritos. Reconocen generar idea y tienen en cuenta las personas a quién se dirige el texto, procurando relacionar unas ideas con otras. Coinciden estos hallazgos con los descubrimientos de Bereiter y Scardamalia (1997) que identifica estrategias elaboradas en adolescentes y adultos donde se establece una dialéctica entre lo que quiere contar, lo que puede ser contado, a quién y de qué modo.

En segundo lugar, los sujetos analizados, cuando escriben, se preocupan de la grafía y la ortografía. Reconocen que revisan sus textos y que realizan cambios, sobre todo, en nexos, en signos de puntuación, caligrafía y ortografía. También cambian partes del texto para mejorar la expresión de las ideas y la estructura del texto. A veces, consultan a otras personas para que revisen sus textos. Coinciden estos hallazgos con el modelo de Berninger y Swanson (1994) que identifican el proceso de la translación (transcripción) es decir, la representación de la escritura en símbolos gráficos. En la translación tiene lugar una serie de operaciones lingüísticas, tales como, aplicación de códigos ortográficos, de normas de cohesión y puntuación y de ejecución grafomotora. También identifican en adolescentes y adultos el proceso de la revisión que, en un principio, se centra en la corrección de palabras o porciones de sentencias, con especial atención a la ortografía y puntuación. Después, esta revisión se extiende a los párrafos y la totalidad del texto, centrándose tanto en el contenido y como en la forma, aplicando diferentes estrategias.

En tercer lugar, los sujetos analizados reconocen utilizar variedad de soportes, destacando el uso del ordenador, en esto coinciden con los estudios de Starke-Meyerring (2009, p. 506) para quien "escribir es tecnología" (citando a Haas, 1996). También, reconocen haber tenido a su alcance recursos humano y materiales de apoyo para sus tareas escritoras, al igual que Almargot y Fayol (2009) que identifica la importancia de los efectos sobre la práctica escritora de los apoyos y el entrenamiento en la escritura.

Los sujetos analizados, en cuarto lugar, admiten que sus escritos pueden ser útiles para los demás, aportando sus ideas y conocimientos, e igualmente reconocen usar la escritura en sus contextos cotidianos. Destacan el logro de intereses propios cuando escriben $\mathrm{y}$, a veces, aplican estrategias propias en la escritura. Estos hallazgos coinciden con el modelo de Hayes (1996) en el que se identifica el contexto de la tarea, como componente que modelan el proceso de escribir. En este contexto se incluye tanto el entorno social como el físico, la motivación, la creatividad...

Sin embargo en la muestra de sujetos analizados, con escasa frecuencias se identifican otros procesos sobre la escritura, señalados por la literatura (apartado 1 de este artículo), tales como: 
1. Seleccionar las ideas o palabras aplicando algún criterio.

2. Aplicar una estructura textual concreta cuando escriben.

3. Realizar cambios en sus textos para lograr la unidad global del texto escrito.

4. Aplicar auto-instrucciones en el proceso escritor.

5. Seleccionar estrategias y códigos lingüísticos según el tipo de texto.

6. Usar los textos para expresar experiencias propias y para expresar valores propios.

7. Usar códigos lingüísticos diferentes en sus escritos.

8. Expresar un auto-concepto positivo en las tareas escritoras.

9. Construido textos de forma colaborativa.

Por último, los sujetos analizados no poseen una Teoría de la Tarea escritora ni una Teoría del Texto, que les permita aplicar estrategias para relacionar el texto con el contextos sociocultural, adaptándose a sus exigencias y condicionamiento. Destaca la ausencia de operaciones de autocontrol en la escritura (concentración y selección emocional).

En vista a las limitaciones encontradas en esta muestra de sujetos, Myhill and Fisher, (2010) destaca que la escritura es una actividad con complejas exigencias que van aumentando, en la medida que el escritor se hace más experto, reclamando gran esfuerzo cognitivo y un gran interés emocional que dinamice la voluntad y autorregule el aprendizaje. Además, la escritura es un proceso socioculturalmente complejo. Cuando los escritores desarrollan un texto, no sólo deben de conocer los patrones de su estructura textual según el género literario, sino que deben prestar atención a cómo, ese género, está mediatizado por el contexto, en el que se desarrolla. Deben ajustarse a los diferentes requerimientos académicos y a las demandas escritoras de los usos tecnológicos y profesionales de la escritura. Y todo esto ocurre sea cual fuere el idioma que se utilice.

En definitiva, si bien los sujetos analizados muestran cierto nivel de competencia procedimental, afectiva y sociocultural de la escritura, evidencian la necesidad de diseñar programas para logra un uso profesional y científico de la misma, que insista en los procesos condicionales escritores. En este sentido en la Universidad de Granada se están diseñando, aplicando y evaluando programas para la enseñanza de la composición escrita científica-profesional. En estos programas se combinan estrategias de aprendizaje para escribir buenos textos de forma independiente (Graham \& Harris, 2005a:26); y el Modelo-CCT (Autor, 2009:213), que utiliza técnicas del trabajo colaborativo e individualizado, apoyadas con aplicaciones tecnológicas de e-learning, usando la Plataforma Moodle. Además, este último modelo didáctico incluye estrategias multilingües de desarrollo escritor, es decir, estrategias que promueven el uso de diferentes lenguas de forma simultánea (español, inglés y otro idioma que el estudiante conozca).

Por último, señalar la principal limitación de esta investigación para su generalización que es el muestreo causal. Sin embargo, nunca fue su pretensión la generalización de las conclusiones. Su intencionalidad fue establecer, con criterios de validez y fiabilidad científica, la transferencia de los hallazgos a otros contextos. Todo ello en coherencia con la línea metodológica de investigación en la escritura más destacada desde Emig (1971), quien introdujo una nueva aproximación para comprender y mejo- 
rar la escritura, partiendo de los conocimientos del escritor y cómo éste lleva a cabo un determinado escrito.

\section{BIBLIOGRAFÍA}

Autor, R. (2000a). Diseño y desarrollo del currículum intercultural. Los valores islámicosoccidentales. Granada: Servicio de publicaciones de la Universidad.

Autor, R. (2000b). Estudio comparativo de valores en jóvenes islámicos y occidentales para una educación intercultural. Revista de Ciencias de la Educación, 182, 129-149.

Autor, R. (2001). Dependencia e independencia de valores por edad y cultura: presupuestos del Currículum Intercultural. Revista de Investigación Educativa, 19 (1), 153-182.

Autor, R. (2006a). La interculturalidad en los procesos de la composición escrita. Investigación en la escuela, 59, 91-102.

Autor, R. (2006b). Desarrollo de la escritura en un contexto multicultural. Una investigación etnográfica. Revista de Investigación Educativa, 24 (2), 305-328

Autor, R. (2009). Desarrollo Metacognitivo y Sociocultural de la Composición Escrita. Interculturalidad y Tecnologías en la enseñanza de la escritura multilingüe. Granada: Nativola.

Autor, R. \& Hunt, C.I. (2010). Strategies, tools and techniques for the developement of writen communication metasociocognitive processes. En N. L. Mertens (Ed.), Writing processes, tools and techniques (pp. 57-74). Nueva York: Nova Science Publishers.

Autor, R. \& Hunt, C. I. (2011). Written communication intercultural model: The social and cognitive model. The International Journal of Interdisciplinary Social Sciences, 6 (1), 19-38.

Autor, R. \& Salvador, F. (2009). Research on cognitive, social and cultural processes of written communication. Cognitive Processig, 10 (3), 263-268.

Alamargot, D., \& Fayol, M. (2009). Modelling the development of written composition. En R. Bear, D. Myhill, M. Nystrand, \& J. Riley (Eds), The sage handbook of writing development (pp. 23-47). Londres: Sage.

Ball, A. (2006). Teaching writing in culturallly diverse classrooms. En C. A. MacArthur, S. Graham, \& J. Fitzgerald (Eds.), Handbook of writing research (pp. 293-310). Nueva York: Guilford Press.

Bardín, L. (1986). Análisis de contenido. Madrid:Akal.

Bear, R. (2000). Developing writing. Londres: Hoodder\&Stoughton.

Bear, R. (2008). Writing development. En R. Autor (Ed.), La Comunicación Escrita: Cognición, Multilingüísmo y Tecnologías (pp. 63-87). Granada: Nativola-Asprogrades.

Bear, R., Myhill, D., Nystrand, M., \& Riley, J. (2009). The sage handbook of writing development. Londres: Sage.

Beriter, C., \& Scardamali, M. C. (1997). The psychology of written composition. Nueva York: Lawrence Erlbaum Aassociates.

Berninguer, V. W., \& Swanson, H. L. (1994). Modifying hayes and flower model of skiled writing to explain beginning and developing writing. En E. C. Butterfield (Ed.), Advances in cognition and educacional practice (Vol.2, pp. 57-82). Greenwich, CT: JAI Press.

Bordieu, P. (2003). El oficio del científico. Ciencia y reflexividad. Barcelona: Anagrama, 
Bruning, R., \& Horn, C. (2000). Developing motivation to write. Educational Psychologist, 35 (1), 25-37.

Cabero, J., \& Llorente, M. C. (2007). La interacción en el aprendizaje en red: uso de las herramientas, elementos de análisis y posibilidades educativas. Revista Iberoamericana de Educación a Distancia, 10 (2), 97-123.

Canagarajah S., \& Jerskey, M. (2009). Meeting the needs of advanced multilingual writers. En R. Bear, D. Myhill, M. Nystrand, \& J. Riley (Eds.), The sage handbook of writing development (pp. 472-488). Londres: Sage.

Daly, J. A., \& Wilson, D. A. (1983). Writing apprehension, self-esteem, and personality. Research in the teaching of English, 4 (17), 327-341.

De Beaugrande, R. (1984). Text production: toward a science of composition. Nueva York: Norwood.

Dezin, N. K., \& Lincoln, I. S. (2011). El campo de la investigación cualitativa (Vol. I). Barcelona: Gedisa.

Denzin, N. K. (1970). Research act. A theoretical introduction to sociological methods. Chicago: Aldine.

Dipardo, A., \& Schnack, P. (2004). Expanding the web of meaning: thought and emotion in an intergenerational reading and writing program. Reading Research Quarterly, 1 (39), 14-37.

Dysthe, O. (2007). How a reform affects writing in higher education. Studies in Higher Education, 32 (2), 237-252.

Emig, J. (1971). The composing processes of twelfth graders. Urbana, IL: National Council of Teachers of English.

Fayol, M. (1991). From sentence production to text production: Investigating fundamental processes. European Journal of Psychology of Education, 6 (2), 101-119.

Fidalgo, R., Arias-Gundín, O., García, N., \& Torrance, M. (2010). Cognitive strategic and self-regulated instruction in writing processes. En N. L. Mertens (Ed.), Writing processes, tools and techniques (pp. 129-152). Nueva York: Nova Science Publishers.

Fitzgerald, J. (2006). Multilingüal writing in preschool through12th grade. Tha last 15 year . En C. A. MacArthur, S. Graham, \& J. Fitzgerald (Eds.), Handbook of writing research (pp. 337-353). Nueva York: Guilford Press.

Flower, L. S., \& Hayes, J. R. (1981). A cognitive process theory of writing. College Composition and Communication, 32 (4), 365-387.

Flick, U. (2007). Introducción a la investigación cualitativa. Madrid: Morata.

Graham, S., \& Harris, K. (1999). Making the writting process work: strategies for composition and self-regualtion. Massachusetts: Brookline Books.

Graham, S., \& Harris, K. (2005a). Writing better: Strategies for teaching students with learning difficulties. Baltimore: Brookes Publishing.

Graham, S., \& Harris, K. (2005b). Improving the writing performance of young struggling writers: Theoretical and programmatic research from the center on accelerating student learning. The Journal of Special Education, 39 (1), 19-33.

Goldberg, A., Russell, M., \& Cook, A. (2003). The effect of computers on student writing: a metaanalysis of studies from 1992 to 2002. Journal of Technology, Learning and Assessment, 2 (1), 120-135. 
Gubrium, J., \& Holstein, J. (2000). Analyzing interpretive practice. En N. Dezin \& Y. Lincoln, (Eds.), Handbook of qualitative research (2aㅡ. ed., pp. 487-508). Londres: Sage Publications.

Hayes, J. R. (1996). A new framework for understanding cognition and affect in writing process. En C. M. Levy \& S. R. Ransdell (Eds.), The sciences of writing (pp. 3-30). Hillsdale, NJ: Lawrence Erlbaum Associates.

Harris, K. R., Graham, S., Mason, L. H., \& Saddler, B. (2002). Developing self-regulated writers. Theory into Practice, 41(2), 110-115.

Hodder, I. (2000). The interpretation of documents and material culture. En N. K. Denzin \& Y. S. Lincoln (Eds.), Handbook of qualitative research (pp. 703-717). Londres: Sage Publications.

Kostouli, T. (2009). A sociocultural framework: Writing as social practice. En R. Bear, D. Myhill, M. Nystrand, \& J. Riley (Eds.), The sage handbook of writing development (pp. 98-115). Londres: Sage.

Krapels, A. (1990). A overview of second language writing process research. En B. Kroll (Ed.), Second language writing: Research insights for the classroom (pp. 37-56). Cambridge, UK: Cambridge University Press.

McArthur, CH. (2006). The effects of new technologies on writing and writing processes. En C. A. MacArthur, S. Graham, \& J. Fitzgeral (Eds.), Handbook of writing research (pp. 249-261). Nueva York: Guilford Press.

MacArthur, C. A., Graham, S., \& Fitzgerald, J. (2006). Handbook of writing research. Nueva York: Guilford Press.

Mayorga, M. J., \& Ruiz Baeza, V. M. (2002). Muestreos utilizados en la investigación educativa en España. Revista Electrónica de Investigación y Evaluación Educativa, 8 (2), 1-8.

Matsuda, P. K., Ortmeier-Hooper., C., \& Matsuda, A. (2009). The expansión of second languaje writing. En R. Bear, D. Myhill, M. Nystrand, \& J. Riley (Eds.), The sage handbook of writing development (pp. 457-471). Londres: Sage.

Myhill, D., \& Fisher, R. (2010). Writing development: cognitive, sociocultural, linguistc perspectives. Journal of Research in Reading, 33 (1), 1-3.

Nystrand, M. (2006). The social and historical context for writing reserach. En C. A. MacArthur, S. Graham, \& J. Fitzgerald (Eds.), Handbook of writing research (pp.1127). Nueva York: Guilford Press.

Rentel, V. M., \& King, M. L. (1983). A longitudinal study of coherence in children written narratives. Washington: National Institute of Education.

Salvador, F., \& García A. (2005). Metodología de la investigación. En F. Salvador (Ed.), La expresión escrita de alumnos con necesidades educativas especiales. Procesos cognitivos (pp. 45-70). Archidona: Aljibe.

Scardamalia, M., \& Bereiter, C. (1983). The development of evaluative, diagnostic and remedial capabilities in children's composing. En M. Martlew (Ed.), The psychology of written language: Developmental and educational perspectives (pp. 67-95). Londres: John Wiley.

Schultz, K., \& Fecho, B. (2000). Society's child: social context and writing development. Educational Psychologist, 35 (1), 51-62. 
Sperling, M., \& Freedman S. W. (2001). Research on writing. En V. Richarson (Ed.), Handbook of research on teaching (4⿳亠丷厂 ed., pp. 370-389). Washington: American Educational Research Association.

Starke-Meyerring, D. (2009). The contested materialities of writing in digital environments: Implications for writing development. En R. Bear, D. Myhill, M. Nystrand, \& J. Riley (Eds.), The sage handbook of writing development (pp. 507-525). Londres: Sage.

Fecha de recepción: 6 de marzo de 2012.

Fecha de revisión: 7 de marzo de 2012.

Fecha de aceptación: 30 de septiembre de 2012. 